\title{
Methods for Assessing Familial Aggregation: Family History Measures and Confounding in the Standard Cohort, Reconstructed Cohort and Case-Control Designs
}

\author{
Regina Zimmerman ${ }^{a, c}$ Deb K. Pal ${ }^{\mathrm{e}}$ Adrienne Tin ${ }^{c}$ Habibul Ahsan ${ }^{a}$ \\ David A. Greenberg ${ }^{\text {b-d }}$ \\ a Department of Epidemiology and b Division of Statistical Genetics, Mailman School of Public Health, \\ 'Department of Psychiatry, Columbia University Medical Center, and d New York State Psychiatric Institute, \\ New York, N.Y., USA, e'Department of Clinical Neuroscience, Institute of Psychiatry, King's College London, \\ London, UK
}

\section{Key Words}

Epidemiology · Family history · Computer simulation •

Sensitivity $\cdot$ Specificity $\cdot$ Confounding

\begin{abstract}
Objectives: To test whether case-control-based familial aggregation studies produce estimates of risk to relatives that are inherently biased or confounded by age and family size, and to compare case-control-derived estimates with those from the reconstructed cohort method. In addition, we test if the definition of family history affects the accuracy of results obtained from either design. We use simulated data, which allows us to know the true data origin. Methods: We simulated populations of three generation families. Both a dominant genetic disease and a non-genetic disease were present in the population. We compared the effect estimates from different measures of family history with those derived from the actual genetic cause of disease. Results: Effect estimates from family history measures that used multiple family members were more accurate than those derived from measures based on a single relative. Neither family size nor age of family members defining family history were confounders in the case-control design. Conclusion: The casecontrol and reconstructed cohort designs are equally valid in assessing familial aggregation of disease.
\end{abstract}

Copyright $\odot 2009$ S. Karger AG, Basel

\section{KARGER}

Fax +41613061234 E-Mail karger@karger.ch www.karger.com (c) 2009 S. Karger AG, Basel

0001-5652/09/0683-0201\$26.00/0

Accessible online at:

www.karger.com/hhe

\section{Introduction}

The clustering of disease in close family members may be explained by sharing of either environmental or genetic factors or both. Familial clustering is often attributed to genetic factors when the risk of disease follows a pattern of biological relatedness within a family, or when environmental factors are not believed to be associated with disease. Familial aggregation studies estimate the increased risk of disease in relatives of affected probands, or the risk to an individual given his or her family history. In this context, the family history of disease may be considered a proxy risk factor for a putative susceptibility genotype. However, there is controversy about the validity of certain study designs for assessing familial aggregation, as well as debate about the best measure of family history to use in familial aggregation studies.

In case-control and cohort designs, one or more relatives affected with disease defines the exposure. The person(s) defining exposure may be limited to relatives in one biological relatedness category, e.g. first degree relatives. In the case-control design, the cases and controls are the outcomes, and the disease status in their relatives is the risk factor, a proxy for exposure. In the reconstructed cohort design, the disease status of precisely one relative - the case or control proband - defines family his-

Dept of Clinical Neuroscience, Institute of Psychiatry

King's College London

London SE5 8AF (UK)

Tel. +44 (0) 207848 5435, Fax +44 (0) 207848 5440, E-Mail Deb.Pal@iop.kcl.ac.uk 
Table 1. Characteristics of nine simulated populations

\begin{tabular}{|c|c|c|c|c|c|c|c|}
\hline \multirow{3}{*}{$\begin{array}{l}\text { Specified } \\
\text { disease } \\
\text { prevalence }^{b}\end{array}$} & \multirow{3}{*}{$\begin{array}{l}\text { Number of } \\
\text { families }^{c}\end{array}$} & \multicolumn{6}{|c|}{ Proportion of disease caused by genetic form ${ }^{\mathrm{a}}$} \\
\hline & & \multicolumn{2}{|c|}{ high, 90\% } & \multicolumn{2}{|c|}{ medium, 50\% } & \multicolumn{2}{|c|}{ low, $10 \%$} \\
\hline & & genetic & non-genetic & genetic & non-genetic & genetic & non-genetic \\
\hline $20 \%$ & 12,000 & $18 \%$ & $2 \%$ & $10 \%$ & $10 \%$ & $2 \%$ & $18 \%$ \\
\hline $10 \%$ & 30,000 & $9 \%$ & $1 \%$ & $5 \%$ & $5 \%$ & $1 \%$ & $9 \%$ \\
\hline $1 \%$ & 54,000 & $0.9 \%$ & $0.1 \%$ & $0.5 \%$ & $0.5 \%$ & $0.1 \%$ & $0.9 \%$ \\
\hline
\end{tabular}

a Two forms of disease are generated, one genetic and one 'random' or sporadic. The familial disease distribution in simulated populations is based on a dominant, $50 \%$ penetrant allele. An allele frequency is estimated to produce a disease prevalence contributing to approximately 90,50 or $10 \%$ of the overall disease prevalence in a given population $(20,10,1 \%)$. The random/phenocopies contribute a random 10,50 or $90 \%$ of the overall disease prevalence in a given population. The relative proportions of genetic and non-genetic forms is derived from the Hardy Weinberg equation; if $\mathrm{p}=$ allele frequency, then $1 / 2\left[\mathrm{p}^{2}+2 \mathrm{p}(1-\mathrm{p})\right]=$ Propor- tion of population with penetrant disease due to the dominant gene.

$\mathrm{b}$ The prevalence of disease in the population is specified in the simulation program. The actual prevalences in the samples are remarkably close to $20,10,1 \%$. See results section for details.

${ }^{c}$ Three generation families with nuclear family size distribution equal to that found in the US population in the 2000 census (10.98\% have 1 offspring; $24.77 \%$ have $2 ; 26.24 \%, 3 ; 16.87 \%$, 4 ; $10.24 \%, 5 ; 5.56 \%, 6$; and $5.34 \%$ have 7 offspring [5]. tory [1]. Relatives of cases and controls are 'reconstructed' into exposed and unexposed cohorts among whom the outcome is examined. Effect estimates in the reconstructed cohort compare disease risk among exposed relatives (relatives of the case) to that among unexposed relatives (relatives of the control). The reconstructed cohort is believed to offer a more conservative (lower) estimate of risk compared to the case-control design.

The case-control design is believed to yield a biased risk estimate [2], with age of relatives and family size being viewed as confounding variables in assessing the disease risk estimate. On the other hand, the reconstructed cohort design is thought to show no such biases. However, these sources of bias and confounding have never been explicitly demonstrated. The aim of this paper is to test if these biases actually exist and compare the performance of the case-control and reconstructed cohort designs. Using computer simulated data of a genetically influenced disease, we asked three questions in order to examine differences between estimates from the various designs. (1) Is the difference in effect estimates in the three study designs due to different family history measures or to the design itself? (2) Is the first degree family history a more sensitive measure of an underlying (genetic vs. non-genetic) cause than the one-relative measure used in the reconstructed cohort design? (3) Is family size a confounder of the association between family history and disease in the case-control design? The simu- lated populations were used as sampling frames to select cases, controls and relatives for the analyses described below.

\section{Methods}

Below, we describe the computer simulated individuals, families and disease parameters and the nine populations created as sampling frames for the study. From each population, we sampled three times, once for each study design (case-control, standard cohort, reconstructed cohort). We outline the analysis methods for the case-control, cohort and reconstructed cohort analyses. Lastly we explain how we measured sensitivity and specificity of the family history measure, and how we tested the hypothesis of confounding by family size.

\section{Simulation}

We used a thoroughly tested computer program to simulate families and diseases for genetic studies [3]. The program requires specification of allele frequencies, mode of inheritance, penetrance, frequency of a non-genetic or random form of disease (socalled sporadics or phenocopies) for which every individual had the same risk, family type (nuclear vs. multiple generations), family size distribution and the number of families. Populations of three-generation families were simulated with Hardy-Weinberg equilibrium, which implies: random mating in the population, a constant mutation rate, no selection for or against particular phenotypes, equally viable genotypes at loci, and no admixture [4]. Family size distributions in three-generation families were based on the given parameters and distributions from the US population [5]. Disease was defined as a dichotomous variable, not cen- 
sored age-at-onset. Follow-up of subjects over time was unnecessary to determine disease incidence because all three generations were generated simultaneously.

We generated nine populations, each with a particular combination of disease prevalence and genetic contribution to disease (table 1). Disease prevalence was varied by changing the gene frequency in the simulation program. One set of populations had 54,000 families each with a disease prevalence of $1 \%$. The second set had 30,000 families each with $10 \%$ disease prevalence. The third set had 12,000 families each with $20 \%$ disease prevalence (table 1).

The genetic form of disease was programmed to have dominant inheritance and be $50 \%$ penetrant. The proportion of the genetic contribution to disease in the population was varied by changing the probability of being affected by the random form, the range of risk for an individual for the random form varied between 0.009 and 0.02 . The risk of disease among the gene carriers is always $50 \%$ plus the risk for the random form. Each prevalence set contained three populations with different ratios of genetic/non-genetic disease: (1) a 'HIGH' genetic composition (approximately 90\% genetic, 10\% non-genetic disease); (2) a 'MEDIUM' genetic composition (approximately 50\% genetic, $50 \%$ non-genetic disease); and (3) a 'LOW' genetic composition (approximately $10 \%$ genetic, $90 \%$ non-genetic disease). Thus, the simulated populations included families in which disease did not aggregate, and families in which either genetic or random or both forms of disease aggregated.

\section{Sampling and Statistical Analyses}

Sampling: There were 9 simulated populations, 3 with 12,000 families, 3 with 30,000 families and 3 with 54,000 families. As described above, each family consisted of three generations. Each mating produced a sibship that followed an empirical family size distribution. For the cohort analysis, one random subject was selected from the middle generation from each of the families in the 9 simulated populations, producing cohorts of either 12,000, 30,000 or 54,000 subjects, depending on which of the nine simulated populations were sampled. This procedure was repeated 50 times, producing 50 cohorts, from which we calculated confidence intervals. The case-control study was nested in the cohort. We designated the $12,000,30,000$ and 54,000 cohort subjects as 'cases' if affected and 'controls' if unaffected. For the 50 trials conducted on each of the 9 simulated populations, the number of cases and controls varied depending on the affected status of the cohort subjects.

\section{Analyses}

We conducted three analyses for each of the nine simulated populations.

(1) Cohort Analysis. We randomly selected one subject from the middle generation of each family, without regard to disease status. The exposure status of this subject was then categorized in three ways: (i) the 'true' genetic relative risk, which is the probability of disease among gene carriers (about 50\%) divided by the probability of disease in the absence of gene carriage; (ii) presence or absence of any first degree family history of disease; or (iii) by the disease status of precisely one randomly selected first degree relative, as used in the reconstructed cohort design. Once exposure status was categorized, then we calculated the risk ratio. The risk ratio (RR) is identical to the incidence rate ratio (IRR) be- cause the 'follow-up time' for exposed and unexposed subjects in the simulated data is equal.

(2) Case-Control Analysis. We designated disease-affected subjects from the cohort study as 'cases' in the case-control study. We chose an equal number of controls out of a random sample of the non-affected subjects from the cohort study, although the cases and controls were not matched. The first degree and one-relative family history for cases and controls were based on the disease status of the case and control relatives. A cumulative incidence based odds ratio (OR) was computed because controls were selected from the sample of cohort members after cases were removed. Although this OR provides a good estimate of the RR when the disease is rare [6], the two effect estimates diverge as disease prevalence increases. We therefore derived a risk ratio for comparison with risk ratio measures in the cohort using the probability of disease in the unexposed cohort [7]:

$$
\mathrm{RR}=\frac{\mathrm{OR}}{\left(1-\mathrm{P}_{0}\right)+\left(\mathrm{P}_{0} \times \mathrm{OR}\right)}
$$

where $\mathrm{P}_{0}$ is the probability of disease among the unexposed.

(3) Reconstructed Cohort Analysis. Cases, controls and relatives were the same in the reconstructed cohort analysis as those used in the case-control analyses. The one difference was that the relatives defined outcome, and the subjects themselves defined exposure, i.e. the reverse of the case-control definitions. The family history definition is limited to the disease status of one relative, the case or control. We computed the risk ratio from the risk of disease among the reconstructed exposed cohort (relatives of cases) and the risk of disease among the reconstructed unexposed cohort (relatives of controls). The confidence intervals around the $\mathrm{RR}$ estimate are falsely narrow because of the correlated risk between relatives.

These three analyses were repeated for fifty samples each drawn from the nine simulated populations. For each scenario, we computed the means and confidence intervals of the fifty effect estimates for each exposure measure.

\section{Sensitivity and Specificity of the Family History Measures}

We can compare the familial RR with the genetic RR because we know the 'true' gene carrier status for each individual in these simulated populations. We randomly selected five of the fifty samples for sensitivity and specificity calculations. Sensitivity was defined as the proportion of gene carrying subjects classified as having a family history of disease. Specificity was defined as the proportion of non-gene-carrying subjects classified as not having a family history. Mean sensitivity and specificity were computed across the five samples.

\section{Confounding by Age and Family Size}

We examined whether age or family size fulfill the definition of a confounder, i.e. a factor that is associated with the exposure of interest as well as a determinant of the outcome [6]. We then sought evidence that family size was a confounder by comparing family size by family history status and disease status of study subjects. Results were presented as crude and adjusted (for family size) ORs. We also tested for possible family-size-related bias by stratifying case and control data by family size. 
Table 2. Risk ratios by family history definition and study design

\begin{tabular}{|c|c|c|c|c|c|c|c|}
\hline & \multirow{2}{*}{$\begin{array}{l}\text { Exposure } \\
\text { definition }^{\text {a }}\end{array}$} & \multicolumn{2}{|l|}{ High $^{b}$} & \multicolumn{2}{|c|}{ Medium $^{b}$} & \multicolumn{2}{|l|}{ Low $^{b}$} \\
\hline & & $\mathrm{RR}^{\mathrm{c}}$ & mean CI & $\mathrm{RR}^{\mathrm{c}}$ & $\mathrm{CI}$ & $\mathrm{RR}^{\mathrm{c}}$ & CI \\
\hline \multicolumn{8}{|c|}{$20 \%$ Disease prevalence } \\
\hline & Genotype & 25.17 & $21.48-29.49$ & 5.64 & $5.40-5.90$ & 3.29 & $3.16-3.42$ \\
\hline \multirow[t]{2}{*}{ FDR } & cohort & 3.56 & $3.19-3.98$ & 1.82 & $1.67-1.98$ & 1.12 & $1.08-1.17$ \\
\hline & case control $^{\mathrm{d}}$ & 3.57 & $3.21-3.95$ & 1.83 & $1.65-2.02$ & 1.07 & $0.96-1.19$ \\
\hline \multirow[t]{3}{*}{1 relative } & cohort & 1.88 & $1.75-2.03$ & 1.54 & $1.43-1.67$ & 1.11 & $1.07-1.16$ \\
\hline & case control $^{\mathrm{d}}$ & 1.89 & $1.71-2.07$ & 1.56 & $1.40-1.73$ & 1.09 & $0.97-1.22$ \\
\hline & recon. cohort & 1.82 & $1.75-1.89$ & 1.51 & $1.45-1.58$ & 1.08 & $1.04-1.13^{\mathrm{e}}$ \\
\hline \multicolumn{8}{|c|}{$10 \%$ Disease prevalence } \\
\hline & Genotype & 48.60 & $42.88-55.09$ & 9.78 & $9.20-10.39$ & 6.16 & $5.68-6.67$ \\
\hline \multirow[t]{2}{*}{ FDR } & cohort & 6.42 & $5.99-6.86$ & 2.24 & $2.11-2.39$ & 1.19 & $1.12-1.27$ \\
\hline & case control $^{\mathrm{d}}$ & 6.41 & $5.88-6.97$ & 2.24 & $2.06-2.43$ & 1.19 & $1.10-1.29$ \\
\hline \multirow[t]{3}{*}{1 relative } & cohort & 3.14 & $2.95-3.34$ & 2.14 & $1.98-2.30$ & 1.24 & $1.12-1.36$ \\
\hline & case control $^{\mathrm{d}}$ & 3.12 & $2.81-3.46$ & 2.11 & $1.87-2.37$ & 1.25 & $1.09-1.43$ \\
\hline & recon. cohort & 3.01 & $2.88-3.15$ & 2.08 & $1.99-2.18$ & 1.22 & $1.16-1.28^{\mathrm{e}}$ \\
\hline \multicolumn{8}{|c|}{$1 \%$ Disease prevalence } \\
\hline & Genotype & 456.53 & $329.28-632.95$ & 110.01 & $92.23-131.20$ & 53.12 & $40.37-69.90$ \\
\hline \multirow[t]{2}{*}{ FDR } & cohort & 57.22 & $48.45-67.24$ & 12.19 & $10.35-14.33$ & 2.07 & $1.60-2.66$ \\
\hline & case control $^{\mathrm{d}}$ & 59.86 & $39.21-88.45$ & 12.72 & $8.55-18.70$ & 2.16 & $1.41-3.32$ \\
\hline \multirow[t]{3}{*}{1 relative } & cohort & 26.74 & $22.27-31.82$ & 12.42 & $9.59-15.95$ & 3.20 & $1.95-5.23$ \\
\hline & case control $^{\mathrm{d}}$ & 34.82 & $12.62-76.37$ & 16.65 & $5.67-48.33$ & 4.53 & $1.29-16.69$ \\
\hline & recon. cohort & 27.23 & $18.87-39.39$ & 13.13 & $9.31-18.54$ & 3.09 & $2.11-4.51^{\mathrm{e}}$ \\
\hline \multicolumn{4}{|c|}{$\begin{array}{l}\text { a Genotype: Subjects having one or both alleles predisposing } \\
\text { them to disease ( } 50 \% \text { penetrance with that predisposing allele); } \\
\text { FDR: Subjects having one or more affected first-degree relatives } \\
\text { classifies them as family history positive. Otherwise they are fam- } \\
\text { ily history negative; one-relative family history measures is com- } \\
\text { parable to family history measure used in the Reconstructed co- } \\
\text { hort. Study subjects having one randomly selected FDR affected } \\
\text { classifies them as family history positive. Otherwise they are fam- } \\
\text { ily history negative. }\end{array}$} & \multicolumn{4}{|c|}{$\begin{array}{l}{ }^{\mathrm{b}} \text { High, Medium, Low }=\text { Levels of genetic disease distribution } \\
\text { (i.e. high indicates } 90 \% \text { genetic, } 10 \% \text { non-genetic disease, medium } \\
\text { indicates } 50 \%: 50 \% \text { and low } 10 \%: 90 \% \text {. } \\
{ }^{c} \text { Mean Risk Ratio computed from } 50 \text { samples drawn from } \\
\text { each of the } 9 \text { simulated population. } \\
{ }^{\mathrm{d}} \text { ORs are converted to RRs. } \\
{ }^{\mathrm{e}} \text { Confidence intervals are inaccurately narrow due to the cor- } \\
\text { related risk between relatives [10]. }\end{array}$} \\
\hline
\end{tabular}

\section{Results}

We present below the results of analyses including expected and observed frequencies of disease in simulated populations, the effect of multiple exposure definitions on effect estimates in populations with varying disease distributions, and the potential for confounding by family size.

\section{Simulated and Expected Disease Prevalence}

Generally, the simulator produced the desired prevalences. In the three datasets specified to have $20 \%$ disease prevalence (high, medium and low genetic influence scenarios), the actual disease prevalences in the samples were $19.53,19.24$ and $19.49 \%$ respectively. Similarly, in the specified $10 \%$ disease prevalence datasets, the three scenarios showed actual prevalences of 10.01, 9.71 and $9.88 \%$. In the $1 \%$ specified prevalence, samples were 0.98 , 0.98 and $1 \%$ respectively.

\section{Variation of Effect Estimates by Study Design and Exposure Measure}

Table 2 displays effect estimates obtained for the three study designs, and the three exposure measures for the nine prevalence and genetic influence scenarios.

Variation by Study Design. Three study designs were examined: cohort, case-control and reconstructed cohort. There was little variation in effect estimate between the three study designs for most scenarios of disease prevalence and genetic influence. Only when the disease 
Table 3. Mean sensitivity and specificity of family history measures of underlying genetic risk derived from cohort studies

\begin{tabular}{|c|c|c|c|c|c|c|c|c|c|}
\hline \multirow{2}{*}{$\begin{array}{l}\text { FH } \\
\text { Definition }\end{array}$} & \multicolumn{3}{|l|}{ High $^{\mathrm{a}}$} & \multicolumn{3}{|c|}{ Medium $^{\mathrm{a}}$} & \multicolumn{3}{|c|}{ Low $^{\mathrm{a}}$} \\
\hline & $\mathrm{RR}$ & sensitivity $^{\mathrm{b}}$ & specificity $^{\mathrm{b}}$ & $\mathrm{RR}$ & sensitivity ${ }^{\mathrm{b}}$ & specificity $^{\mathrm{b}}$ & $\mathrm{RR}$ & sensitivity ${ }^{\mathrm{b}}$ & specificity $^{\mathrm{b}}$ \\
\hline \multicolumn{10}{|c|}{ 20\% Disease prevalence } \\
\hline FDR & 3.56 & 0.88 & 0.51 & 1.82 & 0.90 & 0.38 & 1.12 & 0.94 & 0.28 \\
\hline 1 relative & 1.88 & 0.32 & 0.88 & 1.54 & 0.35 & 0.85 & 1.11 & 0.38 & 0.81 \\
\hline \multicolumn{10}{|c|}{$10 \%$ Disease prevalence } \\
\hline FDR & 6.42 & 0.85 & 0.71 & 2.24 & 0.86 & 0.60 & 1.19 & 0.87 & 0.51 \\
\hline 1 relative & 3.14 & 0.28 & 0.94 & 2.14 & 0.28 & 0.92 & 1.24 & 0.30 & 0.91 \\
\hline \multicolumn{10}{|c|}{$1 \%$ Disease prevalence } \\
\hline FDR & 57.22 & 0.80 & 0.97 & 12.19 & 0.81 & 0.95 & 2.07 & 0.72 & 0.93 \\
\hline 1 relative & 26.74 & 0.22 & 0.99 & 12.42 & 0.23 & 0.99 & 3.2 & 0.18 & 0.99 \\
\hline
\end{tabular}

$\mathrm{FDR}=$ First degree relative $\mathrm{RR}=$ risk ratio.

${ }^{a}$ High, Medium, Low = levels of genetic disease (i.e. high indicates 90\% genetic, $10 \%$ non-genetic disease, medium indicates $50 \%$ : 50\% and low 10\%:90\%.

${ }^{\mathrm{b}}$ Mean sensitivities and specificities of each family history measure computed on 5 samples taken from each simulated population.

prevalence was low (1\%) did the estimates vary somewhat. In this particular scenario, and only when one relative was used to measure exposure, the case-control design yielded an estimate of risk about one-third higher than either of the cohort designs. This elevated risk estimate obtained across high, medium and low genetic influence scenarios.

Variation by Exposure Definition. In all scenarios studied, the effect estimates derived from family history measures vastly underestimate the true risk computed from genotype status. Effect estimates derived from family history measures capture disease aggregation regardless of whether disease has genetic or non-genetic (random) cause. Nevertheless, these proxy measures of exposure yield estimates of risk between three and twenty-five times less than the true genetic relative risk. The difference between these effect estimates and true genetic relative risk is at a minimum at high disease prevalence and when the genetic influence on disease is low but the disease prevalence is high. It is at a maximum when the genetic influence on disease is high but the disease prevalence is low.

Estimates derived from the first degree family history measure are closer to the genetic relative risk than those derived from the one-relative measure. This is especially true in populations with a higher disease prevalence and higher proportion of genetic disease. The difference in effect estimates derived from the two family history measures narrows as the overall prevalence declines and pro- portion of genetic disease declines. The effect estimate derived from the reconstructed cohort is larger than that derived from the case-control study in just one of our scenarios: when disease is rare (1\%) and the proportion of genetic disease is at its lowest.

\section{Sensitivity and Specificity of Family History Measures}

The sensitivity and specificity of first degree and onerelative family history measures was compared against the true genotype status (table 3). The first degree family history measure had superior sensitivity in all combinations of disease prevalence and genetic disease studied. Conversely, the one-relative family history had superior specificity in all scenarios. Specificity, and less so sensitivity, varied by disease prevalence and the proportion of genetic disease. As sensitivity and specificity increase, familial RR estimates become closer to genetic RRs.

However, higher sensitivity does not guarantee an estimate that is closer to the genetic RR. This is because of the relationship between the disease prevalence, the frequency of gene-carriers and risk of disease. As disease prevalence decreases, the frequency of gene-carriers decreases and thus the likelihood of carrying a gene in family history positive families decreases - regardless of the proportion of genetic disease. The less frequent the gene, the more 'sensitive' are risks of disease, derived from family history measures, to changes in specificity.

In these low prevalence disease scenarios, the specificity of the one-relative family history measure is high- 
Table 4. Crude and adjusted (for family size) risk ratios derived from cohort and case-control studies in each simulated population scenario

\begin{tabular}{|c|c|c|c|c|c|c|}
\hline & \multicolumn{2}{|c|}{$\mathrm{High}^{\mathrm{a}}$} & \multicolumn{2}{|c|}{ Medium $^{\mathrm{a}}$} & \multicolumn{2}{|c|}{ Low $^{\mathrm{a}}$} \\
\hline & $\mathrm{RR}^{\mathrm{b}}$ & $\mathrm{CI}$ & $\mathrm{RR}^{\mathrm{b}}$ & $\mathrm{CI}$ & $\mathrm{RR}^{\mathrm{b}}$ & $\mathrm{CI}$ \\
\hline \multicolumn{7}{|c|}{$20 \%$ Disease prevalence } \\
\hline \multicolumn{7}{|c|}{ FDR } \\
\hline \multicolumn{7}{|l|}{ Cohort } \\
\hline Crude & 3.21 & $2.93-3.49$ & 1.82 & $1.67-1.98$ & 1.08 & $1.00-1.18$ \\
\hline Adjusted & 3.41 & $3.12-3.72$ & 1.91 & $1.75-2.08$ & 1.10 & $1.00-1.20$ \\
\hline \multicolumn{7}{|l|}{ Case-control } \\
\hline Crude & 3.20 & $2.88-3.53$ & 1.83 & $1.65-2.02$ & 1.09 & $0.98-1.20$ \\
\hline Adjusted & 3.44 & $3.10-3.81$ & 1.92 & $1.72-2.13$ & 1.10 & $0.98-1.22$ \\
\hline \multicolumn{7}{|l|}{1 relative } \\
\hline \multicolumn{7}{|l|}{ Cohort } \\
\hline Crude & 1.81 & $1.69-1.94$ & 1.54 & $1.43-1.67$ & 1.09 & $0.99-1.19$ \\
\hline Adjusted & 1.81 & $1.69-1.94$ & 1.54 & $1.43-4.67$ & 1.09 & $0.99-1.19$ \\
\hline \multicolumn{7}{|l|}{ Case-control } \\
\hline Crude & 1.81 & $1.65-1.99$ & 1.56 & $1.40-1.73$ & 1.08 & $0.96-1.20$ \\
\hline Adjusted & 1.81 & $1.65-1.99$ & 1.56 & $1.40-1.73$ & 1.08 & $0.96-1.20$ \\
\hline \multicolumn{7}{|c|}{ 10\% Disease prevalence } \\
\hline \multicolumn{7}{|c|}{ FDR } \\
\hline \multicolumn{7}{|l|}{ Cohort } \\
\hline Crude & 6.42 & $5.99-6.86$ & 2.24 & $2.11-2.39$ & 1.19 & $1.12-1.27$ \\
\hline Adjusted & 6.93 & $6.47-7.41$ & 2.40 & $2.25-2.55$ & 1.22 & $1.14-1.30$ \\
\hline \multicolumn{7}{|l|}{ Case-control } \\
\hline Crude & 6.41 & $5.88-6.97$ & 2.24 & $2.06-2.43$ & 1.19 & $1.10-1.29$ \\
\hline Adjusted & 7.02 & $6.43-7.65$ & 2.38 & $2.19-2.59$ & 1.21 & $1.11-1.32$ \\
\hline \multicolumn{7}{|l|}{1 relative } \\
\hline \multicolumn{7}{|l|}{ Cohort } \\
\hline Crude & 3.14 & $2.95-3.34$ & 2.14 & $1.98-2.30$ & 1.24 & $1.12-1.36$ \\
\hline Adjusted & 3.14 & $2.95-3.34$ & 2.14 & $1.98-2.30$ & 1.24 & $1.12-1.36$ \\
\hline \multicolumn{7}{|l|}{ Case-control } \\
\hline Crude & 3.12 & $2.81-3.46$ & 2.11 & $1.87-2.37$ & 1.25 & $1.09-1.43$ \\
\hline Adjusted & 3.12 & $2.81-3.46$ & 2.11 & $1.87-2.37$ & 1.25 & $1.09-1.43$ \\
\hline \multicolumn{7}{|c|}{ 1\% Disease prevalence } \\
\hline \multicolumn{7}{|c|}{ FDR } \\
\hline \multicolumn{7}{|l|}{ Cohort } \\
\hline Crude & 57.22 & $48.45-67.24$ & 12.19 & $10.35-14.33$ & 2.07 & $1.60-2.66$ \\
\hline Adjusted & 63.11 & $53.41-74.12$ & 12.99 & $10.99-15.33$ & 2.08 & $1.61-2.70$ \\
\hline \multicolumn{7}{|l|}{ Case-control } \\
\hline Crude & 59.86 & $39.21-88.45$ & 12.72 & $8.55-18.70$ & 2.16 & $1.41-3.32$ \\
\hline Adjusted & 70.47 & $45.61-104.42$ & 13.25 & $8.87-19.54$ & 2.18 & $1.41-3.36$ \\
\hline \multicolumn{7}{|l|}{1 relative } \\
\hline Cohort & & & & & & \\
\hline Crude & 26.74 & $22.27-31.82$ & 12.42 & $9.59-15.95$ & 3.20 & $1.95-5.23$ \\
\hline Adjusted & 26.74 & $22.27-31.82$ & 12.41 & $9.59-15.95$ & 3.20 & $1.94-5.23$ \\
\hline Case-control & & & & & & \\
\hline Crude & 34.82 & $12.62-76.37$ & 16.65 & $5.67-48.33$ & 4.53 & $1.29-16.69$ \\
\hline Adjusted & 34.84 & $12.62-76.40$ & 16.63 & $5.67-48.26$ & 4.57 & $1.30-16.85$ \\
\hline
\end{tabular}

${ }^{a}$ High, Medium, Low = levels of genetic disease distribution (i.e. high indicates $90 \%$ genetic, $10 \%$ non-genetic disease, medium indicates 50\%:50\% and low 90\%:10\%).

${ }^{\mathrm{b}}$ Mean RR computed from 50 samples taken from simulated population. 
est - because more non-gene carriers are being (correctly) classified family history negative; conversely, sensitivity of the one-relative family history is at its worst. Although sensitivity of the first degree family history is consistently greater in all scenarios, when the gene and random disease are rare, hence disease is rare, the effect estimates derived from the one-relative family history measure are slightly larger than those derived from the first degree relative (FDR) family history measure (table 2, lower right). However, in these same scenarios where the onerelative family history measure is larger than the FDRfamily history measure, neither family history measure results in an effect estimate that approximates the genetic RR.

\section{Confounding by Familial Characteristics in Familial}

Aggregation Studies

The number and age of relatives are both associated with exposure (family history), but neither is a determinant of the disease outcome. Therefore, neither age nor family size meets the definition of a potential confounder for the association between family history and disease [6]. In our simulated data, the number of relatives was associated with family history classification (t test; $\mathrm{p}<$ 0.0001). However, number of relatives did not differ by disease status of study subject. We found no appreciable differences in the odds ratios after adjusting for family size computed in either cohort or case-control analyses (table 4). We also did additional simulations in which we stratified the case and control data by family size. Within strata, there was no bias in the results.

\section{Discussion}

Our results show that effect estimates derived from the case-control design are not inherently biased. In familial aggregation studies, effect estimates vary by family history definition and not by study design. Neither family size nor age fulfil the criteria for confounding factors. The scenarios simulated in this investigation are limited to a single genetic component using a dominant mode of inheritance with $50 \%$ penetrance. The true relative risk was constant within each scenario. Although in reality disease susceptibility is likely to vary within and between families, more complex scenarios for disease susceptibility and environmental causes are unlikely to change our findings.

Khoury and Flanders demonstrated an increasing effect size in the case-control design when the family size in their simulated populations increased [2]. They interpreted this inflation as bias, but it was not bias. In fact the family history measure was changing for the case-control design (based on multiple relatives), while the family history definition (based on the affectedness status of one individual relative), and hence effect estimate, in the reconstructed cohort remained constant. The difference in estimate between the two designs is therefore attributable not to bias but to changes in measurement of exposure.

\section{How Do the Two Measures of Family History \\ Compare to the True Genetic Risk?}

Neither measure serves as a good proxy to genetic risk, but their performance depends on the prevalence of disease as well as the proportion of the disease caused by the genetic form. When disease prevalence is high and the genetic form is common compared to the random form, the first degree relative family history measure is more sensitive [8]. Khoury and Flanders simulated high prevalence disease for their study. This may explain why they found estimates derived from the case-control study, using the FDR family history measure, were consistently larger than those resulting from the reconstructed cohort, using the one-relative family history measure [2]. On the other hand, as the proportion of genetic disease form decreases (increasing the proportion of random disease regardless of overall prevalence), the sensitivity of both family history measures increases slightly. At the same time, the specificity of both family history measures decreases and so the difference between the effect estimates derived from the two measures narrows. Thus, in populations where disease is rare and less aggregated in families, the one-relative family history measure results in effect estimates that more closely reflect the true genetic risk. In populations where familial aggregation is high, family history measures based on multiple family members yield more accurate estimates of the underlying genetic risk.

Neither family size nor age fulfill the conceptual criteria for a confounder, and we have tested this quantitatively for family size. The reconstructed cohort design therefore does not seem to offer an intrinsic advantage over the case-control design in terms of bias or confounding. One possible advantage of the case-control design is the ability to select multiple family members to define exposure, which may lead to an effect estimate closer to the true risk under certain circumstances of prevalence and genetic contribution to disease. Overall however, the choice of design, and the control of error, should be considered in general terms. For example, while our simula- 
tions showed no bias due to family size, it assumed that the family size distributions were the same among case and control data. Systematic error could be introduced if the investigator selects controls whose families differed by size or age from cases or if the disease under study affects the family size or life expectancy of family members. Ascertainment, parity and fertility may also affect risk estimates.

Since no combination of study design or family history measure offers accurate estimates of the true genetic risk, one may argue that the choice of case-control versus reconstructed cohort, or FDR versus multiple relatives to measure family history, is to some degree moot. Given this circumstance, the investigator should be free to adopt the measures and design that best suit the available data to assess familial aggregation. However, if estimating the true genetic risk is not the investigator's primary goal, then using different $\mathrm{FH}$ measures may have implications for counseling using empiric genetic risks.

In the case-control design, where cases and controls are the outcomes and the disease status of the (multiple) relatives is the risk factor, the estimate of familial aggre- gation is useful in settings when a consultand wants to know his or her disease risk given his or her family history (type I relative risk) [9]. Type II relative risks, calculated when the disease status of one relative is considered a risk factor, can be used to quantify the risk of disease to relatives of an affected individual. Type II relative risks will become increasingly important as more risk mutations are identified in genetically influenced diseases.

In conclusion, the case-control design is not inherently biased and neither age nor family size confound the risk estimate. Differences in results between designs are attributable to different FH measures. Neither design approximates the true genotypic risk well. The one-relative measure is useful for counseling or screening relatives of affected individuals.

\section{Acknowledgements}

Funding to support the preparation of this manuscript was received from T32 MH65213, NS047530, NS27941, MH48858, DK31775, P30 ES09089 and P42 ES10349 from the National Institutes of Health.

\section{References}

1 Susser E, Susser M: Familial aggregation studies. A note on their epidemiologic properties. Am J Epidemiol 1989;129:23-30.

2 Khoury MJ, Flanders WD: Bias in using family history as a risk factor in case-control studies of disease. Epidemiology 1995;6:511519.

3 Greenberg DA, MacCluer JW, Spence MA, Falk CT, Hodge SE: Simulated data for a complex genetic trait (problem 2 for GAW11): how the model was developed, and why. Genet Epidemiol 1999;17:S449-459.
4 Thompson MS, McInnes R, Willard H: Genes in Populations; in Thompson MS, McInnes RR, Willard HR (eds): Genetics in Medicine. W.B. Saunders Company, 1991, pp 95-108.

5 U.S.Census. U.S. Census Bureau CSF, 2: http://factfinder.census.gov/, 2000.

6 Rothman KJ, Greenland S: Precision and Validity of Studies; in Rothman KJ, Greenland S (eds): Modern Epidemiology. Philadelphia, Lippincott-Raven, 1998, pp 115-134.

7 Zhang J, Yu KF: What's the relative risk? A method of correcting the odds ratio in cohort studies of common outcomes. JAMA 1998;280:1690-1691.
8 Kelsey JL, Whittemore A, Evans AS, Thompson WD: Measurement Error; in Kelsey JL, Whittemore A, Evans AS, Thompson WD (eds): Methods in Observational Epidemiology. New York, Oxford University Press, 1996, pp 341-363.

\9 Baglietto L, Jenkins MA, Severi G, Giles GG, Bishop DT, Boyle P, Hopper JL: Measures of familial aggregation depend on definition of family history: meta-analysis for colorectal cancer. J Clin Epidemiol 2006;59:114-124.

10 Liang KY, Pulver AE: Analysis of case-control/family sampling design. Genet Epidemiol 1996;13:253-270. 